International Journal of Pure and Applied Mathematics

Volume 84 No. 4 2013, 435-450

ISSN: 1311-8080 (printed version); ISSN: 1314-3395 (on-line version)

url: http://www.ijpam.eu

doi: http://dx.doi.org/10.12732/ijpam.v84i4.13

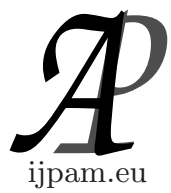

\title{
ON A METHOD FOR CONSTRUCTING SOLUTIONS OF DIFFERENTIAL EQUATIONS OF FRACTIONAL ORDER WITH A HADAMARD TYPE OPERATOR
}

\author{
Batirkhan Turmetov ${ }^{1}$, Kanat Shinaliyev ${ }^{2} \S$ \\ Department of Mathematics \\ International Kazakh-Turkish \\ University named after A. Yasawi \\ Turkestan, 161200, REPUBLIC OF KAZAKHSTAN
}

\begin{abstract}
In this paper we investigate a method for constructing solutions of differential equations of fractional order with Hadamard type derivative. The basis of this method is the construction of systems concerning the operator of fractional differentiation with Hadamard type derivative. We define new classes of special functions generalizing the Mittag-Leffler functions, and related to the differential equations of fractional order. Properties of these functions and their applications to solutions of fractional order differential equations are established. Analytical solutions of differential equations of fractional order are found as a series. Moreover, we consider normed systems of non-homogeneous differential equations of fractional order.
\end{abstract}

AMS Subject Classification: 35J40, 35J05

Key Words: fractional derivative, Hadamard operator, normed system, operator method

\section{Introduction}

Let $0<a<b<\infty, \alpha>0$. For a given $\alpha$ the following expression is called an

Received: January 21, 2013

(C) 2013 Academic Publications, Ltd.

$\S$ Correspondence author url: www.acadpubl.eu 
integration operator of $\alpha$ order in the sense of Hadamard[1]:

$$
I_{a}^{\alpha}(f)(t)=\frac{1}{\Gamma(\alpha)} \int_{a}^{t}\left(\ln \frac{t}{\tau}\right)^{\alpha-1} \frac{f(\tau)}{\tau} d \tau, t>a .
$$

Furthermore, we put $I_{a}^{0}(f)(t) \equiv f(t)$.

Let $\delta=t \frac{d}{d t}, \delta^{k}=\delta\left(\delta^{k-1}\right), k=1,2, \ldots$

Then for $\alpha \in(m-1, m], m=1,2, \ldots$ the following expressions are called differentiation operators of $\alpha$ in the sense of, respectively, Hadamard and HadamardCaputo, see [1]:

$$
\begin{aligned}
& { }_{H} D_{a}^{\alpha}[f](t)=\delta^{m}\left(I_{a}^{m-\alpha} f\right)(t), \\
& { }_{H C} D_{a}^{\alpha}[f](t)=I_{a}^{m-\alpha}\left(\delta^{m} f\right)(t)
\end{aligned}
$$

Let $0 \leq \beta \leq 1, m-1<\alpha \leq m, m=1,2, \ldots$.Then operator

$$
D_{a}^{\alpha, \beta}(f)(t)=I^{\beta(m-\alpha)} \delta^{m} I^{(1-\beta)(m-\alpha)}(f)(t)
$$

is called differentiation operator of $\alpha$ order and $\beta$ type (see $[2,3]$ ). It is clear that if $\beta=0$ then the operator $D_{a}^{\alpha, \beta}$ coincides with ${ }_{H} D_{a}^{\alpha}$, and when $\beta=1$ with the operator ${ }_{H C} D_{a}^{\alpha}$. Note that properties and applications of the operator $D_{a}^{\alpha, \beta}$ when $0<\alpha \leq 1,0 \leq \beta \leq 1$ have been studied in [2].

In this paper we study a method for constructing solutions of differential equations of the following form:

$$
\left(D_{a}^{\alpha, \beta}-\lambda\right)^{n} y(t)=h(t) .
$$

where $\lambda \in R, n=1,2, \ldots$. This method was studied for the differential equations of integer order in [4], and for the equations of fractional order with Riemann-Liouville type operator in $[5,6]$. The basis of this method is the construction of normed systems for the operators $D_{a}^{\alpha, \beta}$ and $D_{a}^{\alpha, \beta}-\lambda$.

Consider definition of the normed systems [4]. Let $L_{1}$ and $L_{2}$ be linear operators given in a linear space of functions $X$. An infinitely system of functions $f_{k}(x) \in X, k=1,2, \ldots$, where any finite subsystem is linear independent, is called $f$-normed concerning $\left(L_{1}, L_{2}\right)$ with base $f_{0}(x)$, if everywhere in this domain the following equality holds:

$$
L_{1} f_{0}(x)=f(x), L_{1} f_{k}(x)=L_{2} f_{k-1}(x), k=1,2, \ldots
$$

A particular case of this definition is the case when $L_{2}$ is an identity operator. In this case, instead of normalizability on the pair of operators $\left(L_{1}, L_{2}\right)$ 
we will talk about normalizability on one operator $L_{1}$. If $f(x)=0$, then the system $f_{k}(x) \in X, k=1,2, \ldots$ is called 0 -normed or just normed [4].

An important property of the normed system is the following: if $\left\{f_{k}(x)\right\}$ is a system of functions $f$-normed concerning $\left(L_{1}, L_{2}\right)$ in the domain $\Omega$ such that the series $y(x)=\sum_{k=0}^{\infty} f_{k}(x)$ converges, and admits termwise application of operators $L_{1}$ and $L_{2}$, then the function $y(x)$ in the domain $\Omega$ is a solution of the equation:

$$
\left(L_{1}-L_{2}\right) y(x)=f(x) .
$$

It is easy to check that if $\left\{f_{k}(x)\right\}$ is a system of functions $f$-normed concerning $L_{1}$ in the domain $\Omega$, and the following equalities hold:

$$
L_{1} L_{2} f_{k}=L_{2} L_{1} f_{k}, k=1,2, \ldots
$$

Then the system of functions $\left\{L_{2}^{k} f_{k}(x)\right\}$ is $f$ - normed concerning $\left(L_{1}, L_{2}\right)$ in the domain $\Omega$. Therefore, to use the basic property of the normed system of functions during the construction of solutions of equation $\left(L_{1}-L_{2}\right) y(x)=$ $f(x)$ it is necessary to find $f$-normed in $\Omega$ system of functions concerning the operator $L_{1}$.

\section{Construction of Normed System for Homogenous Equation}

Let $0 \leq \beta \leq 1, m-1<\alpha \leq m, m=1,2, \ldots, \gamma=(1-\beta)(m-\alpha)$. Introduce the coefficients:

$$
C(\alpha, \beta, s, 0)=1, C(\alpha, \beta, s, i)=\frac{\Gamma(i \alpha+s-\delta+1)}{\Gamma(s-\delta+1)}, i \geq 1,
$$

where $s=0,1, \ldots, m-1$. Let us consider some properties of the operators $I_{a}^{\alpha}$ and $D_{a}^{\alpha, \beta}$.

Lemma 1. Let $s=0,1, \ldots, m-1, m \geq 1$, then

$$
\delta^{m}\left(\ln \frac{t}{a}\right)^{s}=0
$$

Proof. It is obvious that $\delta\left(\ln \frac{t}{a}\right)^{0}=0$ and $\delta\left(\ln \frac{t}{a}\right)=1$. Then for all $s \geq 1$ :

$$
\delta\left(\ln \frac{t}{a}\right)^{s}=s \cdot\left(\ln \frac{t}{a}\right)^{s-1} .
$$


Further, by induction it easy follows that

$$
\delta^{k}\left(\ln \frac{t}{a}\right)^{s}=s \cdot(s-1) \cdot \ldots \cdot(s-k+1)\left(\ln \frac{t}{a}\right)^{s-k}, s \geq k
$$

and

$$
\delta^{k}\left(\ln \frac{t}{a}\right)^{s}=0, \text { if } k>s .
$$

Consequently, for all $s=0,1, \ldots, m-1$ equality (2) holds. Lemma is proved.

Lemma 2. Let $\mu>-1$ and $f(t)=\left(\ln \frac{t}{a}\right)^{\mu}$. Then

$$
I_{a}^{\alpha}(f)(t)=\frac{\Gamma(\mu+1)}{\Gamma(\alpha+\mu+1)}\left(\ln \frac{t}{a}\right)^{\alpha+\mu} .
$$

Proof. By definition of the operator $I_{a}^{\alpha}$ we have

$$
\begin{gathered}
I_{a}^{\alpha}(f)(t)=\frac{1}{\Gamma(\alpha)} \int_{a}^{t}\left(\ln \frac{t}{\tau}\right)^{\alpha-1}\left(\ln \frac{\tau}{a}\right)^{\mu} \frac{d \tau}{\tau}= \\
=\frac{1}{\Gamma(\alpha)} \int_{a}^{t}\left(\ln \frac{t}{a}-\ln \frac{\tau}{a}\right)^{\alpha-1}\left(\ln \frac{\tau}{a}\right)^{\mu} d \ln \frac{\tau}{a}= \\
=\frac{1}{\Gamma(\alpha)} \int_{0}^{1}(1-\xi)^{\alpha-1} \xi^{\mu} d \xi\left(\ln \frac{t}{a}\right)^{\alpha+\mu}=\frac{\Gamma(\mu+1)}{\Gamma(\alpha+\mu+1)}\left(\ln \frac{t}{a}\right)^{\alpha+\mu} .
\end{gathered}
$$

Lemma is proved.

Lemma 3. Let $\mu>m-1$ and $f(t)=\left(\ln \frac{t}{a}\right)^{\mu}$. Then

$$
D_{a}^{\alpha, \beta}(f)(t)=\frac{\Gamma(\mu+1)}{\Gamma(\mu+1-\alpha)}\left(\ln \frac{t}{a}\right)^{\mu-\alpha}
$$

Proof. Let $\mu>m-1$. Due to the equality (4) for the functions $I_{a}^{\gamma}(f)(t)$ we get

$$
I_{a}^{\gamma}(f)(t)=\frac{\Gamma(\mu+1)}{\Gamma(\mu+1+\gamma)}\left(\ln \frac{t}{a}\right)^{\mu+\gamma}
$$


Then by the equality (3) we have

$$
\begin{gathered}
\delta^{m} I_{a}^{\gamma}(f)(t)=\frac{\Gamma(\mu+1)}{\Gamma(\mu+1+\gamma)} \delta^{m}\left(\ln \frac{t}{a}\right)^{\mu+\gamma}= \\
=\frac{\Gamma(\mu+1)}{\Gamma(\mu+1+\gamma)}(\mu+\gamma) \ldots(\mu+\gamma-m+1)\left(\ln \frac{t}{a}\right)^{\mu+\gamma-m}= \\
=\frac{\Gamma(\mu+1)}{\Gamma(\mu+1+\gamma-m)}\left(\ln \frac{t}{a}\right)^{\mu+\gamma-m} .
\end{gathered}
$$

Further,

$$
I_{a}^{\beta(m-\alpha)}\left(\ln \frac{t}{a}\right)^{\mu+\gamma-m}=\frac{\Gamma(\mu+\gamma+1-m)}{\Gamma(\mu+\gamma+1-m+\beta(m-\alpha))}\left(\ln \frac{t}{a}\right)^{\mu+\gamma-m+\beta(m-\alpha)} .
$$

Since $\gamma+\beta(m-\alpha)=m-\alpha$, then

$$
\begin{gathered}
D_{a}^{\alpha, \beta}(f)(t)=I_{a}^{\beta(m-\alpha)} \delta^{m} I_{a}^{\gamma}(f)(t)= \\
=\frac{\Gamma(\mu+1)}{\Gamma(\mu+1+\gamma-m)} \frac{\Gamma(\mu+1+\gamma-m)}{\Gamma(\mu+1-\alpha)}\left(\ln \frac{t}{a}\right)^{\mu-\alpha}=\frac{\Gamma(\mu+1)}{\Gamma(\mu+1-\alpha)}\left(\ln \frac{t}{a}\right)^{\mu-\alpha}
\end{gathered}
$$

Lemma is proved.

Corollary 1. Let $\mu=k \alpha+s-\gamma, k=1,2, \ldots, s=0,1, \ldots, m-1$ and $f(t)=\left(\ln \frac{t}{a}\right)^{\mu}$. Then

$$
D_{a}^{\alpha, \beta}(f)(t)=\frac{\Gamma(k \alpha+s-\gamma+1)}{\Gamma((k-1) \alpha+s-\gamma+1)}\left(\ln \frac{t}{a}\right)^{k \alpha+s-\gamma-\alpha} .
$$

Lemma 4. Suppose that $f_{i}(t)=\frac{1}{C(\alpha, \beta, s, i)}\left(\ln \frac{t}{a}\right)^{\alpha i+s-\gamma}, i=0,1, \ldots$ Then $f_{i}(t)$ for any $s=0,1, \ldots, m-1$ forms 0 -normed system of the functions concerning the operator $D_{a}^{\alpha, \beta}$.

Proof. Let $i \geq 1$. Then,using (6) and definition of the coefficients $C(\alpha, \beta, s, i)$, we get

$$
\begin{gathered}
D_{a}^{\alpha, \beta} f_{i}(t)=\frac{\Gamma(i \alpha+s-\gamma+1)}{\Gamma((i-1) \alpha+s-\gamma+1)} \cdot \frac{\Gamma(s+1-\gamma)}{\Gamma(\alpha i+s-\gamma+1)}\left(\ln \frac{t}{a}\right)^{\alpha i+s-\gamma-\alpha}= \\
=\frac{\Gamma(s+1-\gamma)}{\Gamma((i-1) \alpha+s-\gamma+1)}\left(\ln \frac{t}{a}\right)^{(i-1) \alpha+s-\gamma}=
\end{gathered}
$$




$$
=\frac{1}{C(\alpha, \beta, s, i-1)}\left(\ln \frac{t}{a}\right)^{(i-1) \alpha+s-\gamma}=f_{i-1}(t) .
$$

If $i=0$, then, by the equality (4), we have:

$$
I_{a}^{\gamma}\left(\ln \frac{t}{a}\right)^{s-\gamma}=\frac{\Gamma(s-\gamma+1)}{\Gamma(s-\gamma+1+\alpha)}\left(\ln \frac{t}{a}\right)^{s} .
$$

Since

$$
\delta^{m}\left(\ln \frac{t}{a}\right)^{s}=0, s=0,1, \ldots, m-1,
$$

then

$$
D_{a}^{\alpha, \beta}\left(\ln \frac{t}{a}\right)^{s-\gamma}=0
$$

Lemma is proved.

Consider the function:

$$
\Phi_{\alpha, \beta, s}^{p}(t)=\sum_{i=p}^{\infty} \lambda^{i-p}\left(\begin{array}{c}
i \\
p
\end{array}\right) \frac{t^{\alpha i+s-\delta}}{C(\alpha, \beta, s, i)},
$$

where

$$
p=0,1, \ldots, \quad\left(\begin{array}{c}
i \\
p
\end{array}\right)=\frac{i !}{p !(i-p) !} .
$$

Introduce the following spaces:

$$
C_{\gamma, \ln }[a, b]=\left\{y(t):\left(\ln \frac{t}{a}\right)^{\gamma} \cdot y(t) \in C[a, b]\right\},
$$

and

$$
C_{\gamma}^{\mu}[a, b]=\left\{y(t) \in C_{\gamma, \ln }[a, b]: D_{a}^{\alpha, \beta} y(t) \in C_{\mu, \ln }[a, b]\right\}, \mu>0 .
$$

It is obvious that the spaces $C_{\gamma, \ln }[a, b]$ and $C_{\gamma, \ln }^{\mu}[a, b]$ are linear. The basic properties of the functions $\Phi_{\alpha, \beta, s}^{p}(\lambda, t)$ are presented in the following theorem:

Theorem 1. Let $m-1<\alpha \leq m, 0 \leq \beta \leq 1, s=0,1, \ldots, m-1$. Then $\Phi_{\alpha, \beta, s}^{p}(\lambda, t) \in C_{\gamma, \ln }^{\gamma}[a, b]$, and for all $p=0,1, \ldots$, forms 0-normed system concerning operator $D_{a}^{\alpha, \beta}$ i.e. the following equalities hold:

$$
\begin{gathered}
\left(D_{a}^{\alpha, \beta}-\lambda\right) \Phi_{\alpha, \beta, s}^{0}(\lambda, t)=0, \\
\left(D_{a}^{\alpha, \beta}-\lambda\right) \Phi_{\alpha, \beta, s}^{p}(\lambda, t)=\Phi_{\alpha, \beta, s}^{p-1}(\lambda, t), \quad p \geq 1 .
\end{gathered}
$$


Proof. Let $p=0$. Then the function $\Phi_{\alpha, \beta, s}^{0}(\lambda, t)$ can be presented as a form:

$$
\Phi_{\alpha, \beta, s}^{0}(\lambda, t)=\left(\ln \frac{t}{a}\right)^{-\gamma} \sum_{i=0}^{\infty} \frac{\lambda^{i}}{C(\alpha, \beta, s, i)}\left(\ln \frac{t}{a}\right)^{\alpha i+s}=\left(\ln \frac{t}{a}\right)^{-\gamma} y_{1}(t),
$$

where

$$
y_{1}(t)=\sum_{i=0}^{\infty} \frac{\lambda^{i}}{C(\alpha, \beta, s, i)}\left(\ln \frac{t}{a}\right)^{\alpha i+s} .
$$

It is known that for a gamma function the following asymptotical estimate holds:

$$
\Gamma(z+1)=\sqrt{2 \pi z}\left(\frac{z}{e}\right)^{z}\left\{1+O\left(\frac{1}{z}\right)\right\}, \quad z \rightarrow \infty .
$$

Then it is easy to show that the series (9 converges for $t \in[a, b]$, and the function $y_{1}(t) \in C[a, b]$. Consequently, $\Phi_{\alpha, \beta, s}^{0}(\lambda, t) \in C_{\gamma}[a, b]$. Using operator $D_{a}^{\alpha, \beta}$ to the function $\Phi_{\alpha, \beta, s}^{0}(\lambda, t)$, and taking account that the system $f_{i}(t)=$ $\frac{1}{C(\alpha, \beta, s, i)}\left(\ln \frac{t}{a}\right)^{\alpha i+s}$ forms 0-normed system concerning operator $D_{a}^{\alpha, \beta}$, we receive

$$
\begin{gathered}
D_{a}^{\alpha, \beta}\left[\Phi_{\alpha, \beta, s}^{0}\right](\lambda, t)=\sum_{i=1}^{\infty} \frac{\lambda^{i}}{C(\alpha, \beta, s, i-1)}\left(\ln \frac{t}{a}\right)^{(i-1) \alpha+s}= \\
=\sum_{i=0}^{\infty} \frac{\lambda^{i+1}}{C(\alpha, \beta, s, i)}\left(\ln \frac{t}{a}\right)^{\alpha i+s}=-\lambda \Phi_{\alpha, \beta, s}^{0}(\lambda, t) .
\end{gathered}
$$

Therefore, $D_{a}^{\alpha, \beta}\left[\Phi_{\alpha, \beta, s}^{0}\right](\lambda, t) \in C_{\gamma}^{\gamma}[a, b]$, and it satisfies equality (7). Now let $p \geq 1$. We use operator $D_{a}^{\alpha, \beta}$ to the function $\Phi_{\alpha, \beta, s}^{p}(\lambda, t)$, and similarly as in the case $p=0$, we get

$$
\begin{aligned}
& D_{a}^{\alpha, \beta}\left[\Phi_{\alpha, \beta, s}^{p}\right](\lambda, t)=\sum_{i=p}^{\infty} \lambda^{i-p}\left(\begin{array}{c}
i \\
p
\end{array}\right) D_{a}^{\alpha, \beta} f_{i}(t)= \\
& =\sum_{i=p}^{\infty} \lambda^{i-p}\left(\begin{array}{c}
i \\
p
\end{array}\right) \frac{1}{C(\alpha, \beta, s, i)}\left(\ln \frac{t}{a}\right)^{\alpha(i-1)+s-\delta} .
\end{aligned}
$$

Further, in the last expression by changing the summation index and using the equality:

$$
\left(\begin{array}{l}
i+1 \\
p
\end{array}\right)-\left(\begin{array}{l}
i \\
p-1
\end{array}\right)=\left(\begin{array}{l}
i \\
p
\end{array}\right) \text { and }\left(\begin{array}{l}
p \\
p
\end{array}\right)-\left(\begin{array}{l}
p-1 \\
p-1
\end{array}\right)=0
$$


we obtain

$$
\begin{gathered}
D_{a}^{\alpha, \beta} \Phi_{\alpha, \beta, s}^{p}(\lambda, t)-\Phi_{\alpha, \beta, s}^{p-1}(\lambda, t)= \\
=\sum_{i=p-1}^{\infty} \frac{\lambda^{j-(p-1)}}{C(\alpha, s, j)}\left[\left(\begin{array}{l}
i+1 \\
p
\end{array}\right)-\left(\begin{array}{l}
i \\
p-1
\end{array}\right)\right]\left(\ln \frac{t}{a}\right)^{\alpha j+s-\delta}= \\
=\sum_{i=p-1}^{\infty} \frac{\lambda^{j-(p-1)}}{C(\alpha, s, j)}\left(\begin{array}{l}
i \\
p
\end{array}\right)\left(\ln \frac{t}{a}\right)^{\alpha j+s-\delta}=\lambda \Phi_{\alpha, \beta, s}^{p}(\lambda, t)
\end{gathered}
$$

It follows that

$$
\left(D_{a}^{\alpha, \beta}-\lambda\right) \Phi_{\alpha, \beta, s}^{p}(\lambda, t)=\Phi_{\alpha, \beta, s}^{p-1}(\lambda, t), p \geq 1 .
$$

It is obvious that $\Phi_{\alpha, \beta, s}^{p}(\lambda, t) \in C[a, b]$ for all $p=1, \ldots, n-1$, and $\Phi_{\alpha, \beta, s}^{0}(\lambda, t) \in$ $C_{\gamma}[a, b]$. Hence $D_{a}^{\alpha, \beta} \Phi_{\alpha, \beta, s}^{p}(\lambda, t) \in C_{\gamma}^{\gamma}[a, b]$. Theorem is proved.

Corollary 2. Suppose that conditions of theorem 1 hold for all $p=$ $0,1,2, \ldots, n-1$ and $s=0,1, \ldots, m-1$. Then the functions $\Phi_{\alpha, \beta, s}^{p}(\lambda, t)$ satisfy equality (1) when $h(t)=0$.

Let $p=0$. Transform the function $\Phi_{\alpha, \beta, s}^{0}(\lambda, t)$ to the following form:

$$
\begin{gathered}
\Phi_{\alpha, \beta, s}^{0}(\lambda, t)=\Gamma(s-\gamma+1) \sum_{i=0}^{\infty} \frac{\lambda^{i}}{\Gamma(\alpha i+s-\gamma+1)}\left(\ln \frac{t}{a}\right)^{\alpha i+s-\delta}= \\
=\Gamma(s-\gamma+1)\left(\ln \frac{t}{a}\right)^{s-\delta} \mathrm{E}_{\alpha, s-\gamma+1}\left(\lambda\left(\ln \frac{t}{a}\right)^{\alpha}\right)
\end{gathered}
$$

where

$$
E_{\alpha, \rho}(z)=\sum_{i=0}^{\infty} \frac{z^{i}}{\Gamma(i \alpha+\rho)}
$$

is a Mittag-Leffler type function, see [1].

\section{Construction of Normed System for Non-Homogenous Equation}

Consider the function

$$
h_{0}(t)=\int_{a}^{t}\left(\ln \frac{t}{\xi}\right)^{\alpha-1} E_{\alpha, \alpha}\left(\lambda\left(\ln \frac{t}{\xi}\right)^{\alpha}\right) \frac{h(\xi)}{\xi} d \xi .
$$


In further we assume that $h(t)$ belongs to a class of functions for which all the transactions in question are valid. Let us show that

$$
\left(D_{a}^{\alpha, \beta}-\lambda\right) h_{0}(t)=h(t) .
$$

Consider the case when $\alpha \in(0,1]$. In this case by definition of the operator $D_{a}^{\alpha, \beta}$ we have

$$
D_{a}^{\alpha, \beta} h_{0}(t)=\frac{1}{\Gamma\left(\gamma_{1}\right)} \cdot \int_{a}^{t}\left(\ln \frac{t}{\tau}\right)^{\gamma_{1}-1} \tau \frac{d \tau}{\tau} I_{a}^{\gamma}[h](\tau) \frac{d \tau}{\tau},
$$

where $\gamma_{1}=\beta(1-\alpha)$.

Then

$$
\begin{gathered}
D_{a}^{\alpha, \beta} h_{0}(t)=\frac{1}{\Gamma\left(\gamma_{1}\right)} t \frac{d}{d t}\left\{\int_{a}^{t} \frac{1}{\gamma_{1}}\left(\ln \frac{t}{\tau}\right)^{\gamma_{1}} \frac{d}{d \tau} I_{a}^{\gamma}[h](\tau) d \tau\right\}= \\
=\frac{1}{\Gamma\left(\gamma_{1}\right)} t \frac{d}{d t}\left\{\frac{1}{\gamma_{1}}\left(\ln \frac{t}{\tau}\right)^{\gamma_{1}} I_{a}^{\gamma}[h](\tau) \mid \begin{array}{l}
\tau=t \\
\tau=a
\end{array}+\int_{a}^{t}\left(\ln \frac{t}{\tau}\right)^{\gamma-1} I_{a}^{\gamma}[h](\tau) d\right\}= \\
=\frac{1}{\Gamma\left(\gamma_{1}\right)} t \frac{d}{d t}\left\{-\int_{a}^{t}\left(\ln \frac{t}{\tau}\right)^{\gamma_{1}-1} \frac{1}{\Gamma(\gamma)} \int_{a}^{\tau}\left(\ln \frac{\tau}{\xi}\right)^{\gamma-1} h_{0}(\xi) \frac{d \xi}{\xi} \frac{d \tau}{\tau}\right\}= \\
=\frac{1}{\Gamma\left(\gamma_{1}\right) \Gamma(\gamma)} t \frac{d}{d t} \int_{a}^{t} h_{0}(\xi) \int_{\xi}^{t}\left(\ln \frac{t}{\tau}\right)^{\gamma_{1}-1}\left(\ln \frac{\tau}{\xi}\right)^{\gamma-1} \frac{d \tau}{\tau} \frac{d \xi}{\xi} .
\end{gathered}
$$

Let us study inner integral. We get

$$
\begin{gathered}
\int_{\xi}^{t}\left(\ln \frac{t}{\xi}-\ln \frac{\tau}{\xi}\right)^{\gamma_{1}-\alpha}\left(\ln \frac{\tau}{\xi}\right)^{\gamma-1} d \ln \frac{\tau}{\xi}= \\
=\int_{0}^{1}(1-z)^{\gamma_{1}-1} z^{\gamma-1} d z \cdot\left(\ln \frac{t}{\xi}\right)^{\gamma+\gamma_{1}-1}=\frac{\Gamma\left(\gamma_{1}\right) \Gamma(\gamma)}{\Gamma\left(\gamma+\gamma_{1}\right)}\left(\ln \frac{t}{\xi}\right)^{\gamma+\gamma_{1}-1} .
\end{gathered}
$$


Further, by the presentation of the function $h_{0}(\xi)$ and taking account that $\gamma+\gamma_{1}=1-\alpha$, we have

$$
\begin{gathered}
\int_{a}^{t} h_{0}(\xi)\left(\ln \frac{t}{\xi}\right)^{\gamma+\gamma_{1}-1} \frac{d \xi}{\xi}= \\
=\int_{a}^{t}\left(\ln \frac{t}{\xi}\right)^{-\alpha} \int_{a}^{\xi}\left(\ln \frac{\xi}{\tau}\right)^{\alpha-1} \sum_{i=0}^{\infty} \frac{\lambda^{i}}{\Gamma(\alpha i+\alpha)}\left(\ln \frac{\xi}{\tau}\right)^{\alpha i} \frac{h(\tau)}{\tau} d \tau \frac{d \xi}{\xi}= \\
=\sum_{i=0}^{\infty} \frac{\lambda^{i}}{\Gamma(\alpha i+\alpha)} \int_{a}^{t} h(\tau) \int_{\tau}^{t}\left(\ln \frac{t}{\xi}\right)^{-\alpha}\left(\ln \frac{\xi}{\tau}\right)^{\alpha i+\alpha-1} \frac{d \xi}{\xi} \frac{d \tau}{\tau}= \\
=\sum_{i=0}^{\infty} \frac{\lambda^{i}}{\Gamma(\alpha i+\alpha)} \int_{a}^{t} h(\tau) \frac{\Gamma(1-\alpha) \Gamma(\alpha i+\alpha)}{\Gamma(\alpha i+\alpha+1-\alpha)}\left(\ln \frac{t}{\tau}\right)^{\alpha i} \frac{d \tau}{\tau}
\end{gathered}
$$

Therefore,

$$
\begin{gathered}
D_{a}^{\alpha, \beta} h_{0}(t)=t \frac{d}{d t}\left\{\int_{a}^{t} h(\tau)\left[\sum_{i=0}^{\infty} \frac{\lambda^{i}}{\Gamma(\alpha i+1)}\left(\ln \frac{t}{\tau}\right)^{\alpha i}\right] \frac{d \tau}{\tau}\right\}= \\
=t \frac{h(t)}{t}+\int_{a}^{t} h(\tau)\left[\sum_{i=1}^{\infty} \frac{\lambda^{i}}{\Gamma(\alpha i+1)} \alpha i\left(\ln \frac{t}{\tau}\right)^{\alpha i-1}\right] \frac{d \tau}{\tau}= \\
=h(t)+\int_{a}^{t} h(\tau)\left[\sum_{j=0}^{\infty} \frac{\lambda^{j+1}}{\Gamma(\alpha j+\alpha)}\left(\ln \frac{t}{\tau}\right)^{\alpha j+\alpha-1}\right] \frac{d \tau}{\tau}= \\
=h(t)+\lambda \int_{a}^{t} h(\tau)\left(\ln \frac{t}{\tau}\right)^{\alpha-1} E_{\alpha, \alpha}\left(\lambda\left(\ln \frac{t}{\tau}\right)^{\alpha}\right) \frac{d \tau}{\tau} d \xi=h(t)+\lambda h_{0}(t)
\end{gathered}
$$

Consequently,

$$
D_{a}^{\alpha, \beta} h_{0}(t)=h(t)+\lambda h_{0}(t)
$$

or the same

$$
\left(D_{a}^{\alpha, \beta}-\lambda\right) h_{0}(t)=h(t) .
$$


In general, when $\alpha \in(m-1, m]$ we can present function $D_{a}^{\alpha, \beta} h_{0}(t)$ in the following form:

$$
D_{a}^{\alpha, \beta} h_{0}(t)=I_{a}^{\gamma_{1}} \delta^{m} I_{a}^{\gamma}\left(h_{0}\right)(t)=\frac{1}{\Gamma\left(\gamma_{1}\right)} \int_{a}^{t}\left(\ln \frac{t}{\tau}\right)^{\gamma_{1}-1} \delta g(\tau) \frac{d \tau}{\tau},
$$

where $g(\tau)=\delta^{m-1} I_{a}^{\gamma}\left[h_{0}\right](\tau)$.

Now we study the function $g(\tau)$. To do this, we transform the function $I_{a}^{\gamma}\left[h_{0}\right](\tau)$. By definition of the operator $I_{a}^{\gamma}$, we have

$$
\begin{gathered}
I_{a}^{\alpha} h_{0}(t)=\frac{1}{\Gamma(\gamma)} \int_{a}^{t}\left(\ln \frac{t}{\tau}\right)^{\gamma-1} h_{0}(\tau) \frac{d \tau}{\tau}= \\
=\frac{1}{\Gamma(\gamma)} \int_{a}^{t}\left(\ln \frac{t}{\tau}\right)^{\gamma-1} \int_{a}^{\tau}\left(\ln \frac{\tau}{\xi}\right)^{\alpha-1} E_{\alpha, \alpha}\left(\lambda\left(\ln \frac{\tau}{\xi}\right)^{\alpha}\right) \frac{h(\xi)}{\xi} d \xi \frac{d \tau}{\tau}= \\
=\sum_{i=0}^{\infty} \frac{\lambda^{i}}{\Gamma(\alpha i+\alpha)} \frac{1}{\Gamma(\gamma)} \int_{a}^{t}\left(\ln \frac{t}{\tau}\right)^{\gamma-1} \int_{a}^{\tau}\left(\ln \frac{\tau}{\xi}\right)^{\alpha i+\alpha-1} \frac{h(\xi)}{\xi} d \xi \frac{d \tau}{\tau}= \\
=\sum_{i=0}^{\infty} \frac{\lambda^{i}}{\Gamma(\alpha i+\alpha)} \frac{1}{\Gamma(\gamma)} \int_{a}^{t} h(\xi) \int_{\xi}^{t}\left(\ln \frac{t}{\tau}\right)^{\gamma-1}\left(\ln \frac{\tau}{\xi}\right)^{\alpha i+\alpha-1} \frac{d \tau}{\tau} \frac{d \xi}{\xi}= \\
=\int_{a}^{t}\left[\sum_{i=0}^{\infty} \frac{\lambda^{i}}{\Gamma(\alpha i+\alpha+\gamma)}\left(\ln \frac{t}{\xi}\right)^{\alpha i+\alpha-1+\gamma}\right] \frac{h(\xi)}{\xi} d \xi
\end{gathered}
$$

Further, since

$$
\delta^{m-1}\left(\ln \frac{t}{\xi}\right)^{\alpha i+\alpha-1+\gamma}=(\alpha i+\alpha-1+\gamma) \ldots(\alpha i+\alpha+\gamma-(m-1))
$$

and

$\Gamma(\alpha i+\alpha+\gamma)=(\alpha i+\alpha+\gamma-1) \ldots(\alpha i+\alpha+\gamma-(m-1)) \Gamma(\alpha i+\alpha+\gamma-(m-1))$,

then

$$
D_{a}^{\alpha, \beta} h_{0}(t)=\frac{1}{\Gamma\left(\gamma_{1}\right)} t \frac{d}{d t}\left\{\int_{a}^{t} \frac{1}{\gamma_{1}}\left(\ln \frac{t}{\tau}\right)^{\gamma_{1}} \frac{d}{d \tau} g(\tau) d \tau\right\}=
$$




$$
=\frac{1}{\Gamma\left(\gamma_{1}\right)} t \frac{d}{d t}\left\{\int_{a}^{t}\left(\ln \frac{t}{\tau}\right)^{\gamma_{1}} g(\tau) d \tau\right\} .
$$

Now it is obvious that $\lim _{\tau \rightarrow \infty} g(\tau)=0$. Then by (12) as in the case $\alpha \in(0,1)$ we get

$$
\begin{aligned}
D_{a}^{\alpha, \beta} h_{0}(t) & =\frac{1}{\Gamma\left(\gamma_{1}\right)} t \frac{d}{d t}\left\{\int_{a}^{t} \frac{1}{\gamma_{1}}\left(\ln \frac{t}{\tau}\right)^{\gamma_{1}} \frac{d}{d \tau} g(\tau) d \tau\right\}= \\
& =\frac{1}{\Gamma\left(\gamma_{1}\right)} t \frac{d}{d t}\left\{\int_{a}^{t}\left(\ln \frac{t}{\tau}\right)^{\gamma_{1}} g(\tau) d \tau\right\}
\end{aligned}
$$

Further,

$$
\begin{gathered}
\int_{a}^{t}\left(\ln \frac{t}{\tau}\right)^{\gamma_{1}-1} g(\tau) d \tau= \\
=\sum_{i=0}^{\infty} \frac{\lambda^{i}}{\Gamma(i \alpha+\alpha+\gamma-(m-1))} \int_{a}^{t}\left(\ln \frac{t}{\tau}\right)^{\gamma_{1}-1} \int_{a}^{\tau}\left(\ln \frac{\tau}{\xi}\right)^{\alpha i+\alpha+\gamma-m} \frac{h(\xi)}{\xi} d \xi \frac{d \tau}{\tau}= \\
=\sum_{i=0}^{\infty} \frac{\lambda^{i}}{\Gamma(i \alpha+\alpha+\gamma-(m-1))} \int_{a}^{t} \frac{h(\xi)}{\xi} \int_{\xi}^{t}\left(\ln \frac{t}{\tau}\right)^{\gamma_{1}-1}\left(\ln \frac{\tau}{\xi}\right)^{\alpha i+\alpha+\gamma-m} \frac{d \tau}{\tau} d \xi= \\
=\Gamma\left(\gamma_{1}\right) \sum_{i=0}^{\infty} \frac{\lambda^{i}}{\Gamma(\alpha i+1)} \int_{a}^{t}\left(\ln \frac{t}{\xi}\right)^{\alpha i} \frac{h(\xi)}{\xi} d \xi= \\
=\Gamma\left(\gamma_{1}\right) \int_{a}^{t}\left[\sum_{i=0}^{\infty} \frac{\lambda^{i}}{\Gamma(\alpha i+1)}\left(\ln \frac{t}{\xi}\right)^{\alpha i}\right] \frac{h(\xi)}{\xi} d \xi
\end{gathered}
$$

Consequently,

$$
\begin{gathered}
D_{a}^{\alpha, \beta} h_{0}(t)=t \frac{d}{d t}\left\{\int_{a}^{t}\left[\sum_{i=0}^{\infty} \frac{\lambda^{i}}{\Gamma(\alpha i+1)}\left(\ln \frac{t}{\xi}\right)^{\alpha i}\right] \frac{h(\xi)}{\xi} d \xi\right\}= \\
=h(t)+\int_{a}^{t}\left[\sum_{i=1}^{\infty} \frac{\lambda^{i}}{\Gamma(\alpha i+1)} \alpha i\left(\ln \frac{t}{\xi}\right)^{\alpha i-1}\right] \frac{h(\xi)}{\xi} d \xi=
\end{gathered}
$$




$$
=h(t)+\lambda \int_{a}^{t}\left[\sum_{j=0}^{\infty} \frac{\lambda^{j}}{\Gamma(\alpha j+\alpha)}\left(\ln \frac{t}{\xi}\right)^{\alpha j+\alpha-1}\right\rfloor \frac{h(\xi)}{\xi} d \xi=h(t)+\lambda h_{0}(t) .
$$

So,

$$
\left(D_{a}^{\alpha, \beta}-\lambda\right) h_{0}(t)=h(t) .
$$

Further, suppose $p=0,1, \ldots D_{a}^{\alpha, \beta}-\lambda$ and

$$
E_{\alpha, \alpha}^{p}(\lambda, t)=\sum_{i=p}^{\infty} \lambda^{i-p}\left(\begin{array}{c}
i \\
p
\end{array}\right)\left(\ln \frac{t}{a}\right)^{\alpha i} .
$$

It is obvious that

$$
E_{\alpha, \alpha}^{0}(\lambda, t)=E_{\alpha, \alpha}(\lambda, t)
$$

when $p=0$.

Introduce the function:

$$
h_{p}(t)=\int_{a}^{t}\left(\ln \frac{t}{\xi}\right)^{\alpha-1} E_{\alpha, \alpha}^{p}\left(\lambda,\left(\ln \frac{t}{\xi}\right)^{\alpha}\right) h(\xi) \frac{d \xi}{\xi} .
$$

Theorem 2. Let $m-1<\alpha \leq m, 0 \leq \beta \leq 1, s=0,1, \ldots, m-1$.

Then the functions $h_{p}(t), p=0,1, \ldots$ form $h$-normed system concerning operator, i.e. the following equalities hold:

$$
\begin{gathered}
\left(D_{a}^{\alpha, \beta}-\lambda\right) h_{0}(t)=h(t), \\
\left(D_{a}^{\alpha, \beta}-\lambda\right) h_{p}(t)=h_{p-1}(t), \quad p \geq 1 .
\end{gathered}
$$

Proof. Since when $p=0 E_{\alpha, \alpha}^{0}(\lambda, t)=E_{\alpha, \alpha}(\lambda, t)$ then we have proved equation (13) above. Let $p \geq 1$. As in the case $p=0$ we present function $D_{a}^{\alpha, \beta} h_{p}(t)$ in the form:

$$
D_{a}^{\alpha, \beta} h_{p}(t)=\frac{1}{\Gamma\left(\gamma_{1}\right)} t \frac{d}{d t}\left\{\int_{a}^{t} \frac{1}{\gamma_{1}}\left(\ln \frac{t}{\tau}\right)^{\gamma_{1}} \frac{d}{d \tau} f(\tau) d \tau\right\}
$$

where $\gamma_{1}=\beta(m-\alpha), \quad f(\tau)=\delta^{m-1} I_{a}^{\gamma} h_{p}(\tau)$. Further,

$$
I_{a}^{\alpha} h_{p}(\tau)=\frac{1}{\Gamma(\gamma)} \int_{a}^{\tau}\left(\ln \frac{\tau}{\xi}\right)^{\gamma-1} h_{p}(\xi) \frac{d \xi}{\xi}=
$$




$$
\begin{gathered}
=\frac{1}{\Gamma(\gamma)} \int_{a}^{\tau}\left(\ln \frac{\tau}{\xi}\right)^{\gamma-1} \int_{a}^{\xi}\left[\sum_{i=p}^{\infty} \frac{\lambda^{i-p}}{\Gamma(\alpha i+\alpha)}\left(\begin{array}{c}
i \\
p
\end{array}\right)\left(\ln \frac{\xi}{z}\right)^{\alpha i+\alpha-1} \frac{h(z)}{z} \frac{d \xi}{\xi}=\right. \\
=\sum_{i=p}^{\infty} \frac{\lambda^{i-p}}{\Gamma(\alpha i+\alpha)}\left(\begin{array}{c}
i \\
p
\end{array}\right) \frac{1}{\Gamma(\gamma)} \int_{a}^{\tau} h(z) \int_{z}^{\tau}\left(\ln \frac{\tau}{\xi}\right)^{\gamma-1}\left(\ln \frac{\xi}{z}\right)^{\alpha i+\alpha-1} \frac{d \xi}{\xi} \frac{d z}{z}= \\
=\int_{a}^{\tau}\left[\sum_{i=p}^{\infty} \frac{\lambda^{i-p}}{\Gamma(\alpha i+\alpha+\gamma)}\left(\begin{array}{c}
i \\
p
\end{array}\right)\left(\ln \frac{\tau}{z}\right)^{\alpha i+\alpha-1+\gamma} h(z) \frac{d z}{z}\right.
\end{gathered}
$$

Hence for a function $f(\tau)$ we obtain

$$
f(\tau)=\delta^{m} I_{a}^{\gamma}\left[h_{p}\right](\tau)=\int_{a}^{\tau}\left[\sum_{i=p}^{\infty} \frac{\lambda^{i-p}}{\Gamma(\alpha i+\alpha+\gamma-(m-1))}\left(\ln \frac{\tau}{z}\right)^{\alpha i+\alpha+\gamma-m} h(z) \frac{d z}{z} .\right.
$$

Consequently, integrating by parts in (15), we get

$$
D_{a}^{\alpha, \beta} h_{p}(t)=\frac{1}{\Gamma\left(\gamma_{1}\right)} t \frac{d}{d t}\left\{\int_{a}^{t}\left(\ln \frac{t}{\tau}\right)^{\gamma_{1}-1} f(\tau) \frac{d \tau}{\tau}\right\}
$$

Further, as in the $\operatorname{case} p=0$ we have

$$
\int_{a}^{t}\left(\ln \frac{t}{\tau}\right)^{\gamma_{1}-1} f(\tau) \frac{d \tau}{\tau}=\Gamma\left(\gamma_{1}\right) \int_{a}^{t}\left[\sum_{i=p}^{\infty} \frac{\lambda^{i-p}}{\Gamma(\alpha i+1)}\left(\begin{array}{c}
i \\
p
\end{array}\right)\left(\ln \frac{t}{\xi}\right)^{\alpha i} \frac{h(\xi)}{\xi} d \xi\right.
$$

It means that

$$
\begin{aligned}
& D_{a}^{\alpha, \beta} h_{p}(t)=\int_{a}^{t}\left[\sum_{i=p}^{\infty} \frac{\lambda^{i-p}}{\Gamma(\alpha i)}\left(\begin{array}{l}
i \\
p
\end{array}\right)\left(\ln \frac{t}{\xi}\right)^{\alpha i-1}\right\rfloor \frac{h(\xi)}{\xi} d \xi= \\
& =\int_{a}^{t}\left[\sum_{j=p-1}^{\infty} \frac{\lambda^{j-(p-1)}}{\Gamma(\alpha j+\alpha)}\left(\begin{array}{l}
j+1 \\
p
\end{array}\right)\left(\ln \frac{t}{\xi}\right)^{\alpha j+\alpha-1} \frac{h(\xi)}{\xi} d \xi\right.
\end{aligned}
$$

Then

$$
\begin{gathered}
D_{a}^{\alpha, \beta} h_{p}(t)-h_{p-1}(t)= \\
=\int_{a}^{t}\left\{\sum_{i=p-1}^{\infty}\left[\left(\begin{array}{l}
i+1 \\
p
\end{array}\right)-\left(\begin{array}{l}
i \\
p-1
\end{array}\right)\right] \frac{\lambda^{i-(p-1)}}{\Gamma(\alpha i+\alpha)}\left(\ln \frac{t}{\xi}\right)^{\alpha i+\alpha-1}\right\} \frac{h(\xi)}{\xi} d \xi=
\end{gathered}
$$




$$
=\int_{a}^{t}\left\{\sum_{i=p}^{\infty}\left(\begin{array}{c}
i \\
p
\end{array}\right) \frac{\lambda^{i-(p-1)}}{\Gamma(\alpha i+\alpha)}\left(\ln \frac{t}{\xi}\right)^{\alpha i+\alpha-1}\right\} \frac{h(\xi)}{\xi} d \xi=\lambda h_{p}(t) .
$$

Therefore,

$$
D_{a}^{\alpha, \beta} h_{p}(t)-h_{p-1}(t)=\lambda h_{p}(t)
$$

i.e. for all $p=1,2, \ldots$ the following equality holds:

$$
\left(D_{a}^{\alpha, \beta}-\lambda\right) h_{p}(t)=h_{p-1}(t) .
$$

Theorem is proved. Theorems 1 and 2 imply the following basic statement:

Theorem 3. Let $m-1<\alpha \leq m,, \mathrm{~m}=1,2, \ldots, 0 \leq \beta \leq 1$. Then the function

$$
y(t)=\sum_{s=0}^{m-1} \sum_{p=0}^{n-1} C_{s, p} \Phi_{\alpha, \beta, s}^{p}(\lambda, t)+\int_{a}^{t}\left(\ln \frac{t}{\xi}\right)^{\alpha-1} E_{\alpha, \alpha}^{n-1}\left(\lambda,\left(\ln \frac{t}{\xi}\right)^{\alpha}\right) h(\xi) \frac{d \xi}{\xi}
$$

is a solution of equation (1), where $C_{s, p}$ are constants.

\section{Acknowledgments}

This paper is financial supported by the grant of MES RK No.0713/GF.

\section{References}

[1] A.A. Kilbas, H.M. Srivastava, J.J. Trujillo, Theory and Applications of Fractional Differential Equations, Elsevier, North-Holland (2006).

[2] M.D. Qassim, K.M. Furati, N.-E. Tatar, On a differential equation involving hilfer-Hadamard fractional derivative, Abstract and Applied Analysis (2012), Article ID 391062, 17.

[3] R. Hilfer, Y. Luchko, Z. Tomovski, Operational method the solution of fractional differential equations with generalized Rieman-Lioville fractional derivatives, Fractional Calculus and Applied Analysis, 12, No. 3 (2009), 299-318.

[4] B.A. Bondarenko, Operator Algorithms in Differential Equations, Tashkent, "FAN" (1984), In Russian. 
[5] K.M. Shinaliyev, B.Kh. Turmetov, S.A. Umarov, Fractional operator algorithm method for construction of solutions of fractional order differential equations, Fractional Calculus and Applied Analysis, 15, No. 2 (2012), $267-281$.

[6] B.Kh. Turmetov, K.M. Shinaliyev, On an operator method of construction the solution of differential equations of the fractional order, Vestnik of $L$. Gumilyev Eurasian National University. Series for Natural and Engineering Sciences, 81, No. 2 (2011), 10-17. 